\title{
Gravitational waves from galaxy encounters
}

\author{
Vicent Quilis, ${ }^{1}$ A. César González-García, ${ }^{2,3}$ Diego Sáez, ${ }^{1}$ and José A. Font ${ }^{1}$ \\ ${ }^{1}$ Departament d'Astronomia i Astrofísica, Universitat de València, Dr. Moliner 50, 46100 Burjassot (València), Spain \\ ${ }^{2}$ Instituto de Astrofísica de Canarias, Vía Láctea s/n, La Laguna, 38200, Spain \\ ${ }^{3}$ Departamento de Física Teórica, C-XI, Universidad Autónoma de Madrid, Madrid, 28049, Spain
}

(Received 11 September 2006; revised manuscript received 3 March 2007; published 11 May 2007; publisher error corrected 16 May 2007)

\begin{abstract}
We discuss the emission of gravitational radiation produced in encounters of dark matter galactic halos. To this aim we perform a number of numerical simulations of typical galaxy mergers, computing the associated gravitational radiation waveforms as well as the energy released in the processes. Our simulations yield dimensionless gravitational wave amplitudes of the order of $10^{-13}$ and gravitational wave frequencies of the order of $10^{-16} \mathrm{~Hz}$, when the galaxies are located at a distance of $10 \mathrm{Mpc}$. These values are of the same order as those arising in the gravitational radiation originated by strong variations of the gravitational field in the early Universe, and therefore, such gravitational waves cannot be directly observed by ground-based detectors. We discuss the feasibility of an indirect detection by means of the $B$-mode polarization of the cosmic microwave background (CMB) induced by such waves. Our results show that the gravitational waves from encounters of dark matter galactic halos leave much too small an imprint on the CMB polarization to be actually observed with ongoing and future missions.
\end{abstract}

DOI: 10.1103/PhysRevD.75.104008

PACS numbers: 04.30.Db, 95.35.+d, 98.65.Fz, 98.80.Cq

\section{INTRODUCTION}

Galaxy encounters and interactions have wellestablished observational evidence. Supermassive black holes (SBHs) in the mass range of $10^{6}-10^{9} M_{\odot}$ are firmly believed to exist at the cores of AGNs and quasars. During such interactions gravitational radiation is going to be emitted. As a result, a stochastic background of gravitational waves (GWs) from SBH encounters and coalescence sweeps the cosmos, with wave frequencies of the order of $10^{-6} \mathrm{~Hz}$ or below. The gravitational waves produced by the merger of binary SBHs, in terms of both the amplitude of the signal as well as the frequency range, are amenable to detection by the Laser Interferometer Space Antenna (LISA), becoming one of the most promising anticipated targets with an optimist signal-to-noise ratio of about 1000 [1].

Binary black hole evolution and merger has received considerable numerical attention in recent years through $N$-body simulations (see, e.g. Refs. [2,3] and references therein). Examples include galaxy mergers in which the premerger galaxies contain power-law nuclear cusps and massive particles representing the SBHs. Previous work on the GWs emitted from such galaxy interactions have only considered the radiation produced during the coalescence of the central SBHs [4,5]. Thus far, however, no attention has yet been paid to the gravitational radiation emitted from the bulk of matter of the interacting galaxies, i.e. neglecting the central SBHs, notwithstanding the fact that the mass of the galaxy which lies outside the black hole is typically some orders of magnitude larger than the SBH mass itself. The underlying reason for the absence of investigations in such aspect of galaxy encounters may have to do with the fact that the size of the coalescing objects involved (the whole galaxies) is not compact enough to yield a direct observational measure in the frequency range of the upcoming interferometer LISA.

On the other hand the dominant component accounting for the total mass of the galaxy is the dark matter part. Hence, an interesting question to ask oneself is whether a hypothetical detection of the gravitational radiation emitted from galaxy encounters, modeled as interactions of (collisionless) dark matter halos lacking the SBH at the center, would provide an unambiguous signature of the presence of such elusive matter. If that were the case the detection of those GWs would then provide a smoking-gun signature of yet another traditionally evasive concept in gravitational physics, namely, dark matter.

Our aim in this work is to present and discuss numerical simulations of a reduced but representative sample of such galaxy encounters and to compute the associated gravitational waveforms and luminosities. Building on these results we conjecture about the detectability of such signals by electromagnetic means, namely, through their imprint on the polarization of the cosmic microwave background (CMB hereafter).

The nonlinear evolution of cosmological scalar perturbations as sources of the CMB polarization has been discussed by Ref. [6] using perturbation theory. More recently, the authors of Ref. [7] have reported a related study which focus on the cosmological stochastic GW background produced by idealised cold dark matter halos via power transfer from scalar and vector perturbations to tensor metric modes, during the strongly nonlinear stage of their evolution. Since the nonlinear evolution of such halos occurs on a cosmological timescale, the associated gravitational radiation may be relevant at frequencies comparable to those of the primordial GW which affect the $\mathrm{CMB}$ photons and produce secondary CMB anisotropy and po- 
larization. While the idea behind our work shares the views of Ref. [7], the GWs we analyze arise from galacticlike halos of dark matter, which are substantially more asymmetric. Moreover, our study focus on the particular episode of galactic mergers, where the geometrical features of the process produce GWs with distinctive (burstlike) waveforms and larger amplitudes than those considered by Ref. [7] and therefore, their effects on the CMB polarization are likely to have greater significance.

Our study is further motivated by the prospects of potential experimental measurability. There are a number of missions aimed to detect signatures of GWs (with wavelengths comparable to the size of the Universe) produced by quantum fluctuations of spacetime during inflation, by measuring the weak imprint they leave on the $B$-mode polarization of the CMB. There have already been claims [8] suggesting that the polarization induced on the CMB by relic gravitational waves at large scales can be already measured on the available data from WMAP, a situation which is likely to be improved by the Planck mission. According to Ref. [9] the $B$-mode polarization spectrum of the CMB due to both, primordial (inflation) gravitational waves and also gravitational lensing, is well within the range of detectability of the Planck satellite. In addition, several experiments have been proposed for measuring the polarization ( $E$ and $B$ modes) of the $\mathrm{CMB}$ with higher sensitivity than WMAP (see e.g. Ref. [10]). Specially suited for the detection of $B$-mode polarization induced by relic GWs are the upcoming projects CLOVER [11] and BICEP [12]. Furthermore, among the missions included in NASA's Beyond Einstein program (see UNIVERSE.NASA.GOV) the Cosmic Inflation Probe also appears well suited to investigate the ultralow frequency GWs from the early Universe.

The simulations reported in this paper show that the frequencies of the gravitational waves produced by galaxy mergers $\left(\sim 10^{-16} \mathrm{~Hz}\right)$ match the frequency sensitivity window of the aforementioned future missions (namely the Cosmic Inflation Probe). The amplitude of these GW signals is likely to be of comparable order than those coming from primordial waves originated during the early Universe. However, we estimate that the CMB polarization they produce is weaker than that due to primordial waves. While the imprint of the gravitational radiation from galaxy mergers on the CMB polarization is inaccessible to detection, the gravitational lensing effect associated with gravity perturbations produced in our simulated galaxy mergers could marginally lie within the range of detectability of a number of planned missions designed to measure such polarization. This issue will be discussed elsewhere.

The paper is organized as follows: In Sec. II we describe the galaxy model, the setup of the numerical simulations, and the numerical code used. In Sec. III the basic concepts and equations used to extract gravitational waveforms within the (Newtonian) slow-motion approximation are described. The results of our investigation are presented in Sec. IV. Finally, Sec. V outlines the summary of this research.

\section{GALAXY MODEL AND NUMERICAL APPROACH}

Galaxy mergers are believed to be a formation mechanism for present-day elliptical galaxies [13]. Different formation scenarios have been claimed to form high luminosity boxy ellipticals and low-luminosity disky ellipticals. Boxy ellipticals could be formed through dry mergers of bulge dominated progenitors [14-17] while disky ellipticals could be formed through unequal mass merging of disks with a prominent bulge [18] or by including gas in the initial systems [19].

For the present study we adopt the collisionless approximation as a reasonable approach and simulate the merger of two elliptical galaxies and the merger of two disks with three different configurations (see Table I). The initial conditions for the models described here are taken from the samples studied by Refs. $[14,18]$. We resimulate three key experiments with a larger number of particles. We choose those experiments that produced the largest asymmetries during the merging stages for the remnants with realistic properties as compared to real-life ellipticals.

To build and run the simulations we adopt nondimensional units with $G=1$ for Newton's constant of gravity. We use isotropic spherical Jaffe models [20] as initial conditions for the elliptical galaxies (see Ref. [14] for a detailed description of the algorithm). The projected surface density of such models exhibits a slope that decreases with the radius roughly as $R^{1 / 4}$, which makes it a suitable representation for elliptical galaxies. Jaffe model is characterized by the theoretical half-mass radius $r_{\mathrm{J}}$, which we choose to be equal to 1 . Because a cutoff radius is imposed at $r=10 \times r_{\mathrm{J}}$, the true half-mass radius $r_{1 / 2}$ is 0.82 .

We follow Ref. [21] to build the initial disk galaxy models. Our initial models have a bulge, a disk, and a halo. The initial parameters for these models are identical to models $d b h$ in Ref. [18] but with a larger number of particles. The model with a different mass is a scaled up version of the low mass system following a Tully-Fisherlike relation.

TABLE I. Initial configurations for the models. (1) Model name, (2) type of merger, elliptical-elliptical $(E+E)$ or diskdisk $(S+S)$, (3) Mass ratio, (4) initial orientation of the spin, (5) initial separation, (6) orbit ellipticity, and (7) impact parameter.

\begin{tabular}{lcccccl}
\hline \hline Mod. (1) Type (2) $\frac{M_{1}}{M_{2}}(3)$ & $\left(\theta_{1}, \phi_{1}\right)\left(\theta_{2}, \phi_{2}\right)(4)$ & $r_{i}(5)$ & $e(6)$ & $D(7)$ \\
\hline$M 1$ & $\mathrm{E}+\mathrm{E}$ & $1: 1$ & $(0,0) ;(0,0)$ & 40 & 1 & 0 \\
$M 2$ & $\mathrm{E}+\mathrm{E}$ & $1: 1$ & $(0,0) ;(0,0)$ & 30 & 1 & 4.9 \\
$M 3$ & $\mathrm{~S}+\mathrm{S}$ & $3: 1$ & $(10,-10) ;(70,30)$ & 12.4 & 0.7 & 3.72 \\
\hline \hline
\end{tabular}


For scaling our Jaffe models to elliptical galaxies the set of units is $[M]=M_{\mathrm{J}}=4 \times 10^{11} M_{\odot},[L]=r_{\mathrm{J}}=10 \mathrm{kpc}$, $[T]=2.4 \times 10^{7} \mathrm{yr}$, and finally $[v]=414 \mathrm{~km} / \mathrm{s}$. A set of physical units that match the $d b h$ models to the Milky Way are $[M]=3.24 \times 10^{11} M_{\odot},[L]=14.0 \mathrm{kpc},[T]=4.71 \times$ $10^{7} \mathrm{yr}$, with $[v]=315 \mathrm{~km} / \mathrm{s}$.

Table I reports the orbital parameters of the simulations. We first consider two equal-mass mergers between elliptical galaxies with different impact parameters. Model M1 is a head-on collision on a parabolic orbit, while model M2 is a grazing encounter with a fair impact parameter, the orbital angular momentum is thus larger. Each elliptical galaxy is modeled with $4 \times 10^{5}$ particles for model M1 and with $10^{5}$ particles for run M2. Model M3, on the other hand, is a merger between two disk galaxies with a mass ratio 3:1. Both systems are placed on penetrating prograde orbits. The orbits are elliptical ( $e=0.7$ ) to ensure merging and avoid large computational times. The two disks are placed with their spin slightly coupled with the orbital angular momentum. Each disk galaxy simulation consists of $2.75 \times 10^{5}$ particles for the halo, $1.75 \times 10^{5}$ particles for the disk and $9 \times 10^{4}$ for the bulge. This makes a total of $10^{6}$ particles for the merger remnant. For all models of our sample, after merger is completed the remnants are let to evolve in isolation for 10 half-mass radius crossing times to ensure that the inner parts are close to virial equilibrium.

We have performed the simulations with the parallel version of GADGET [22] using 16 CPU's on the IAC Beowulf Cluster. Each run takes of the order of $5 \times$ $10^{5}$ seconds of CPU time. Independent softening is applied for each particle type. A tolerance parameter of $\theta=0.8$, quadrupole terms, and a variable time step have been used. Energy conservation is satisfactory as variations are kept below the $0.5 \%$ level.

\section{GRAVITATIONAL RADIATION}

The study presented in this paper is focused in galaxy encounters taking place, in a flat Universe, at distances $(D<100 \mathrm{Mpc})$ much smaller than the horizon scale. The galactic systems we consider are far from being relativistic in both senses, special relativity $\left(v / c \leq 10^{-3}\right)$, and general relativity $\left(r_{g} / R \leq 10^{-4}, \quad r_{g}=2 G M / c^{2}\right.$ being the Schwarzschild radius of the object).

Under these conditions the gravitational radiation emitted from the encounters of our galaxy halos can be described by the so-called slow-motion formalism, where the spacetime metric can be linearised in the usual way $\left(g_{\mu \nu}=\right.$ $\eta_{\mu \nu}+h_{\mu \nu}$ ) and the transverse traceless (TT) gauge can be used everywhere outside the system [23]. The spatial components of $h_{\mu \nu}^{\mathrm{TT}}$ are

$$
h_{i j}^{\mathrm{TT}}=\frac{G}{c^{4}} \frac{2}{D} \ddot{I}_{i j}\left(t-\frac{D}{c}\right),
$$

where $I_{i j}$ are the components of the traceless inertial tensor.
The collisionless particle contribution to $I_{i j}$ is given by the following summation extended over all matter particles

$$
I_{i j}=\sum_{n_{p}} m_{p}\left(x_{i} x_{j}-\frac{1}{3} \delta_{i j} x^{2}\right)
$$

$n_{p}$ being the number of particles, $m_{p}$ the mass of each particle, and $\delta_{i j}$ Kronecker's delta.

Outside the galaxies, the relative motion of two neighboring particles $A$ and $B$ moving with the cosmological background (ideal detector) is fully described by the quantities $h_{i j}^{\mathrm{TT}}$. In the TT gauge there is a system of coordinates attached to $A$ in which the coordinate variations of the particle $B$ are $X_{B}^{i}(t)-X_{B 0}^{i}=\frac{1}{2} X_{B 0}^{j}\left[h_{i j}^{\mathrm{TT}}(t)\right]_{A}$, where $X_{B 0}^{i}$ stands for the initial coordinates of the particle $B$ and the quantities $h_{i j}^{\mathrm{TT}}$ are calculated at point $A$. From this formula, it follows that oscillations in the $h_{i j}^{\mathrm{TT}}$ quantities lead to oscillations in the relative position of particles $A$ and $B$ with related frequencies and amplitudes.

In the slow-motion approximation, the gravitational luminosity, $L_{\mathrm{GW}}$, is given by the well-known formula

$$
L_{\mathrm{GW}}=\frac{G}{5 c^{5}} \sum_{i j} \dddot{I}_{i j} \dddot{I}_{i j}
$$

A direct calculation of $L_{\mathrm{GW}}$ based on Eq. (3) is problematic [24] as a result of the noise introduced in the numerical computation of third-order time derivatives. However, as several authors have suggested (see, e.g. Ref. $[25,26]$ ), the second-order time derivatives involved in $\ddot{I}_{i j}$ can be written in terms of the quantities $\ddot{x}$ and $\dot{x}$, which, in its turn, can be expressed in terms of related variables using the equations of motion of the system.

It is well known that, in the case of gravitational waves, the metric perturbation $h_{i j}$ only depends on two independent linear polarization states. Any electromagnetic radiation propagating in a spacetime perturbed from Minkowski by the presence of gravitational radiation, will suffer such polarization itself. A particularly interesting case is represented by the CMB, and it is indeed possible to take advantage of the polarization of the CMB to design strategies to detect gravitational waves $[8,27]$. The case of relic, ultralow frequency GWs has been recently studied in great detail by Ref. [8]. The order of magnitude of the amplitude and frequency corresponding to the present-day primordial GW spectrum is $\sim 10^{-15}-10^{-5}$ and $\sim 10^{-20}-10^{-15}$, respectively, (see Fig. 2 in Ref. [8]).

We will show in the following section that the source investigated in our work, galaxy encounters, leads to values of the GW strain and frequencies which overlap to those arising in strong variations of the gravitational field in the early Universe. 


\section{RESULTS}

\section{A. Dynamics of the encounters}

We begin the discussion of our results by analyzing the morphological features which arise in our simulations of galaxy mergers, deferring for the next section the implications of such dynamics in the emission of gravitational radiation.

Figure 1 displays three snapshots of the three simulated merger encounters. Model M1 (Fig. 1 left column) is a head-on encounter between two equal-mass elliptical galaxies placed on an orbit with parabolic orbital energy. The two galaxies approach each other and there is not much disturbance while they get closer prior to the first pass through the pericenter (which corresponds to the middle row of Fig. 1), apart from a heating of the outer parts. A large fraction of the orbital energy is lost in the first encounter, a number of particles are expelled from the system, becoming unbound and probably forming part of the unbound free floating intergalactic population [28]. These particles carry out a fraction of the orbital energy away leaving the cores of the merging galaxies on a bound orbit. A rapid merging follows next, leading to the forma- tion of a single object which is far from virial equilibrium right after the merging happens. A reorganization of the structure, orbital composition of the stars and shape of the object takes place during some time, leaving behind a prolate nonrotating remnant when the simulation is stopped after the central parts of the remnant are close to virial equilibrium (bottom row of Fig. 1).

Model M2 (Fig. 1 middle column) is an equal-mass encounter between two elliptical galaxies, which follow a parabolic orbit with a large impact parameter. This simulation differs from the previous one in the type of encounter. While for model M1 the encounter was head-on, model M2 corresponds to a grazing encounter. Again, prior to the first pass through the pericenter there is not much evolution in the two galaxies. After the first encounter and due to the acting tidal forces, the galaxies feature extended plumes and broad bridges of particles between the two bodies. Like in model M1, a fraction of the orbital energy is transferred to the particles as kinetic energy. A number of particles acquire positive binding energy and thus escape. After a second pass through the pericenter the galaxies finally merge. The orbital angular momentum of the initial galaxies is transferred as internal angular momen-
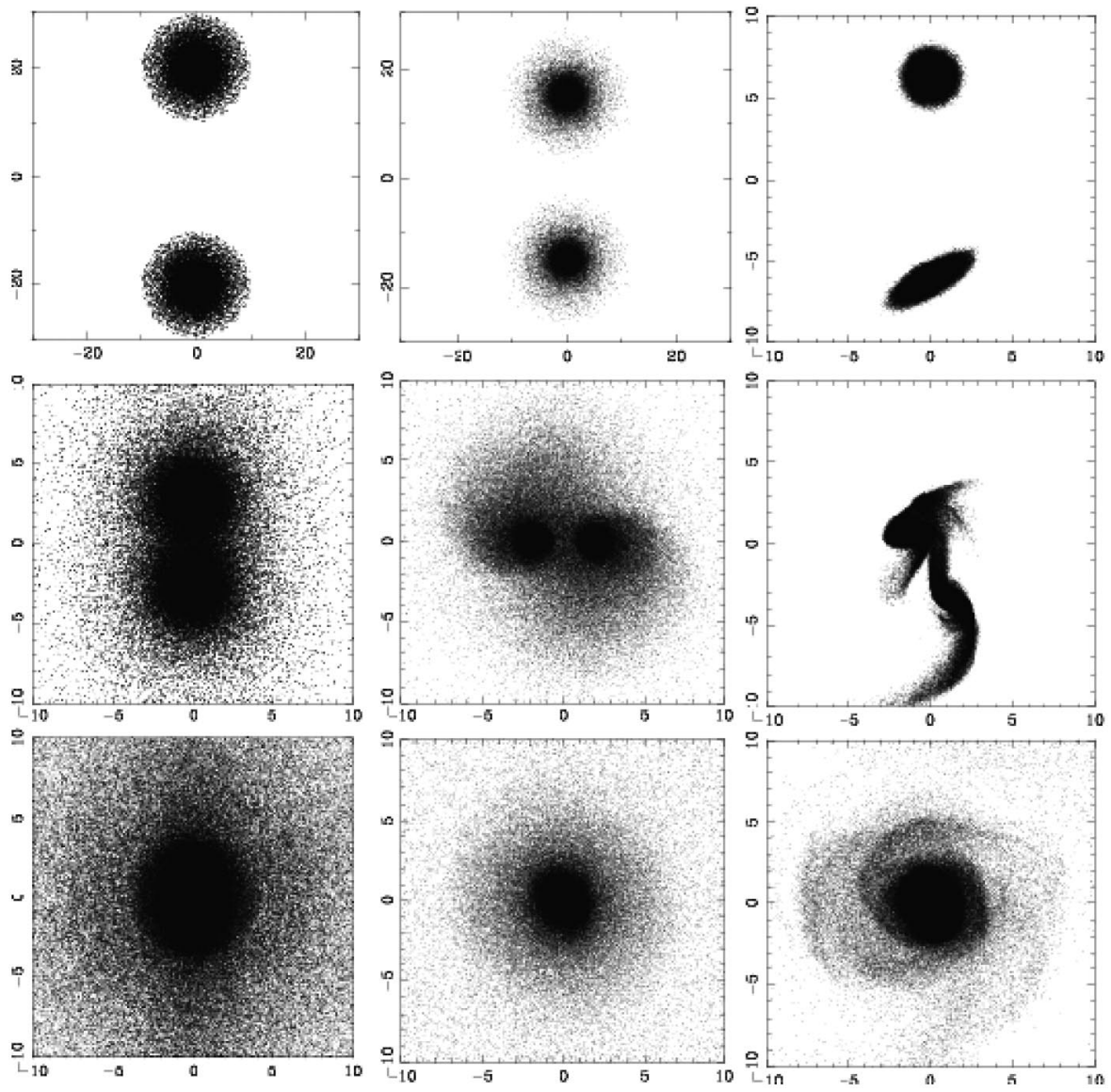

FIG. 1. Dynamical evolution of the three merger simulations as seen from a point of view parallel to the orbital angular momentum. Left column: M1, middle column: M2, right column: M3. Top row initial conditions, middle row right after the first pass through pericenter, bottom row at the end of the simulation. 
tum to the stars of the merged system. As a consequence the final remnant presents a fair amount of rotation and due to the gentle merging orbit the final shape of the object is rather oblate.

These two systems and merging processes give as a result remnants that match the properties found in present-day, high luminosity elliptical galaxies. Therefore, this kind of dry-merger between elliptical galaxies has been proposed as a formation mechanism for present-day, high luminosity ellipticals $[14,16]$.

Model M3 (Fig. 1 right column) corresponds to the collision between two disk-bulge-halo models with their spins partially aligned with the orbital angular momentum. Not much evolution of the disks is seen prior to the first encounter, although some distortion and heating is observed. However, after the first pass through the pericenter (middle row) prominent tails and a bridge between the two central bodies are created due to the spin-orbit coupling. The tidal tails carry away a number of stars that transport a large fraction of the angular momentum to the outer parts. After two more passes through the pericenter new tails are formed and finally the main bodies merge. Shells and ripples are still visible in the outer parts of the remnant after the inner parts have merged (bottom row). The large bulges of the initial systems help to stabilize the disks during the merging stages, as discussed by Ref. [18], so right after the last encounter and the merging a large fraction of the disk particles still have disk kinematics, leaving a clear signature in the merger remnant. Both the orbital angular momentum and the disks spin angular momentum are transferred to the inner parts of the remnant so the kinematics of this system is that of an oblate rotator and the isophotes of the remnant present disky deviations from perfect ellipses. This process has been proposed as a way to build present-day mid- and low-luminosity elliptical galaxies [18,29].

\section{B. Gravitational radiation: waveforms and energetics}

We turn now to describe the gravitational radiation generated in the galaxy encounters discussed in the preceding section. Figure 2 shows the dimensionless GW strain $h$ (only the "+" polarization is plotted) as a function of time for the three models. These waveforms have been computed using Eq. (1). This equation shows that the strain decays linearly with the distance. In order to compute the curves of Fig. 2 we have assumed a distance of $D=$ $10 \mathrm{Mpc}$ to the source, which is compatible with observable galactic systems undergoing merger events (e.g. NGC 4424, The Antennae galaxies NGC 4038/9 or NGC 520) located at few tens of Mpc. We have checked that the amplitude scales with the distance as expected, while the frequency remains unaffected. The mergers we are studying occur on characteristic timescales of a few $10^{9}$ years. Note that the radiation signal reaches its maximum amplitude in such timescales. Therefore, such immense wave-
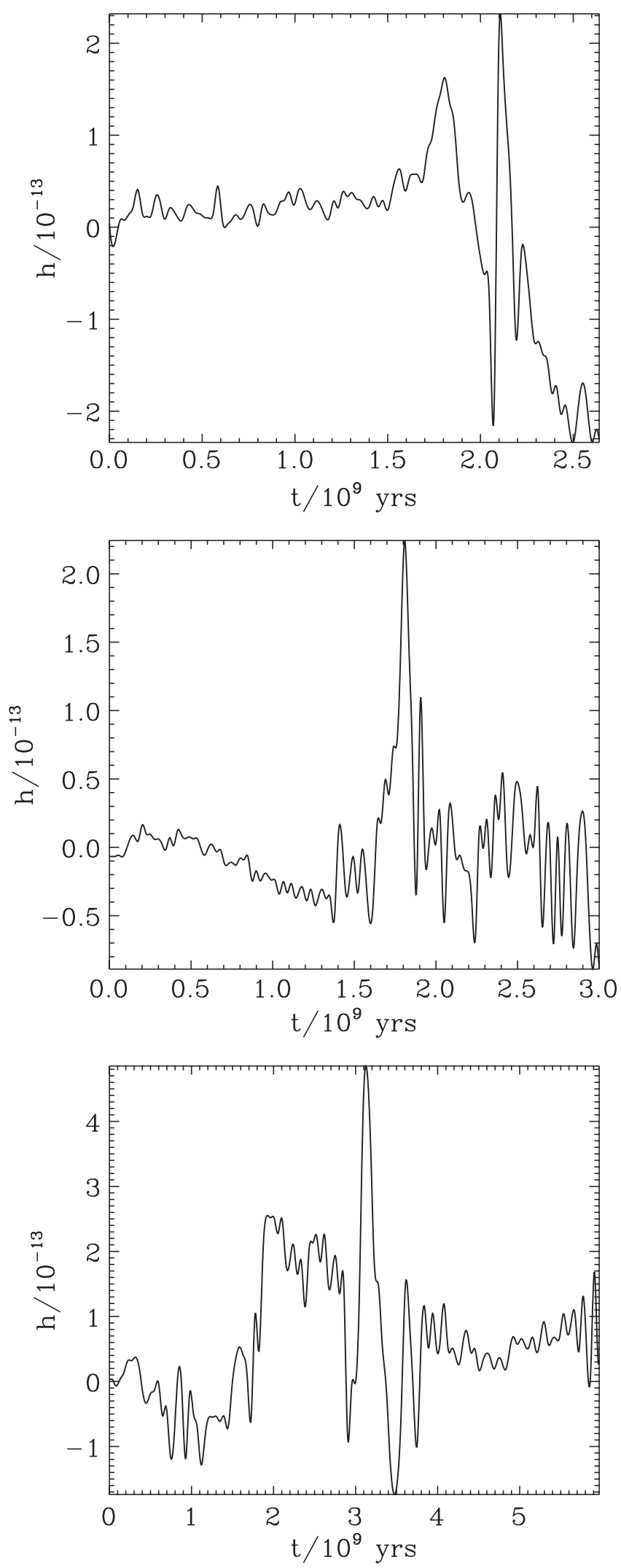

FIG. 2. Gravitational waveform for the three models: M1 (top), M2 (middle), and M3 (bottom). Only the "+" polarization mode is shown. Galaxies are located at $D=10 \mathrm{Mpc}$ from the observer. 
lengths lead to tiny $\mathrm{GW}$ frequencies of the order of $\sim 10^{-16} \mathrm{~Hz}$.

From the very beginning of the simulations there is a non-negligible contribution to the GW signal associated with the internal dynamics of the halos. This is due to the fact that the halos are made of collisionless particle distributions which are not in equilibrium and possess a nonzero quadrupole moment. All three waveforms are dominated by a maximum burstlike signal which is correlated with the final plunge of the two galaxies, taking place at times $\sim 2 \times 10^{9}$ (top), $\sim 1.8 \times 10^{9}$ (middle), and $\sim 3 \times$ $10^{9}$ (bottom) years, for models M1, M2, and M3, respectively. Since the mergers extend considerably in time, even though the final snapshots in Fig. 1 show virialized systems (for their central parts), the waveforms keep memory of the violent events which leads to noticeable variations being still visible in the GW signal by the end of the simulations, particularly for model M1. This effect, however, does not have any implication on the typical signal amplitudes $(h \sim$ $10^{-13}$ ) and frequencies attained.

The different dynamics of the mergers in models M1 and M2 is reflected in the waveform patterns shown in the upper top panels of Fig. 2. In both cases the plunge phase is clearly noticeable in the burst signal. Model M1 corresponds to a head-on collision, a process somehow more violent and shorter than that present in model M2, which describes a nonzero impact parameter encounter leading to a grazing collision. Despite the intrinsically different evolutionary tracks in models M1 and M2, the waveform gross features are similar. This could be explained by the complexity of the scenario, and the way in which the waveforms are computed by adding the GW contribution from each dark matter particle, which may lead to a complex interference pattern. Therefore, the different dynamics in both models is hard to disentangle in the GW signal.

On the other hand model M3 corresponds to a disklike encounter. These system are inherently more aspherical and, from the early phase of the evolution, the signal is stronger than in cases M1 and M2. As a direct consequence of the morphology of the disklike galaxies involved in this encounter, tidal disruptions highly alter the disks for a more extended period of time than in the other two cases. Tidal streams are visible in the middle panel of Fig. 1 for model M3 (after the first pass through the pericenter), which translates in a modulated high-amplitude GW signal during most of the simulation. The signal peaks at $t \sim$ $3.5 \times 10^{9}$ years which, as in the other two cases, corresponds to the plunge event. After this, the signal decays keeping a high amplitude and variability due to the oblate geometry of the final object (see the spiral structure in the bottom panel of Fig. 1 for model M3).

It is worth pointing out that since the values of $h$ and the frequency of the GWs resulting from our simulated galaxy encounters are of the order of those expected from relic GWs (see e.g. Fig. 2 of Ref. [8]), their effect on the CMB polarization is also likely to exist.
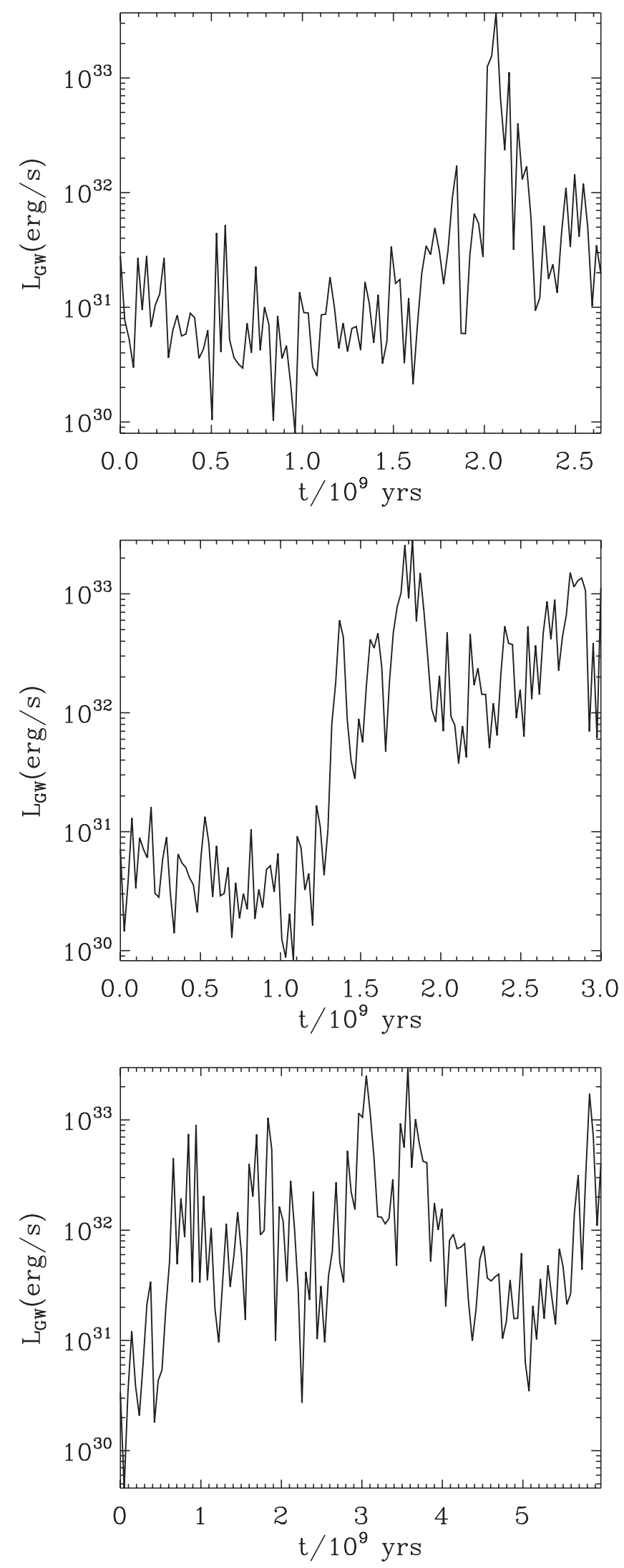

FIG. 3. Gravitational wave luminosity as a function of time for the three models: M1 (top), M2 (middle), and M3 (bottom). 
We note that our results, in terms of both GW frequencies and amplitudes, differ from those of Ref. [7], being slightly larger for the case of galaxy encounters studied here. The explanation is to be found in the fact that [7] study the formation and evolution of isolated, idealized, dark matter halos. No encounters are considered and the contribution to the GW signal comes only from the quiescence evolution of such halos.

On the other hand, we have not included in our simulations the presence of SBHs in the numerical modelling. This has been done on purpose, motivated by the interest in searching for the contribution to the GW signal from the collisionless component of the galaxies, leaving aside the contribution from the SBHs. This has no influence for the signal we are studying since it is well known that binary SBH mergers show chirp waveforms whose frequencies fall within the sensitivity range of LISA [1] and, hence, contribute in a extremely different band of the GW spectrum.

Figure 3 shows the time evolution of the gravitational luminosity of the three models of our sample. The curves are computed using Eq. (3), reducing the order of the time derivatives through the use of the equations of motion. The first thing to mention is the correlation between the maxima of the luminosities and the GW amplitudes. As happens with the signal strain (see Fig. 2) the luminosity in model M3 shows increased modulation than for models M1 and M2. We estimate that the average GW energy emitted during the whole duration of the simulations for all models is $\sim 10^{49} \mathrm{erg}$.

These results show that direct detection of GWs from galaxy mergers is entirely inaccessible to observation. In addition, we note that, unfortunately, gravitational waves from galaxy encounters cannot themselves polarize the $\mathrm{CMB}$ in a significant manner. An estimate based on Ref. [30], which is only valid for a source located in the wave zone (e.g. $100 \mathrm{Mpc}$ ), produces in our case a rotation angle of the polarization vector of the CMB photons $\sim 10^{-13}$ radians, a completely negligible effect. On the other hand, as mentioned in the introduction it has been recently claimed [7] that the distribution of dark matter halos with masses between $5 \times 10^{9} M_{\odot}$ and $10^{15} M_{\odot}$ produces a background of GWs whose effects on the CMB polarization deserve a detailed study. In particular, $B$-mode polarization could be detected in future missions tailored to study this polarization mode. This issue deserves a detailed study which will be presented elsewhere.

\section{SUMMARY}

In this paper we have studied the gravitational waves produced by galaxy encounters, paying attention to the collisionless component of the systems. To the best of our knowledge such an investigation has not been carried out before. For our study we have performed numerical simulations of a sample of representative galaxy encoun- ters, for which we have extracted, using the Newtonian quadrupole formula, the gravitational waveforms, amplitudes and frequencies, as well as the luminosities. The main result of our work has been to find out that GWs produced in galaxy encounters have typical values of the GW strain and frequencies which overlap to those arising in strong variations of the gravitational field in the early Universe. Our simulations confirm that the absence of the central SBH does not affect the GW spectra we have computed, since the signals from binary SBH mergers have very different features, in terms of frequency and amplitude, to the ones presented in this paper.

Recently, a number of missions have been proposed to detect signatures of GWs (with wavelengths comparable to the size of the Universe) produced by quantum fluctuations of spacetime during inflation, by measuring the weak imprint they leave on the polarization of the CMB. Given the fact that the main features of our results could fit in the expected range of detectability of those missions, we have estimated the effects of a close galaxy encounter on the CMB polarization. Thus, we have investigated this possibility to find out whether this effect could add an unexpected source of GW "noise" which may hinder the original target of the experiments, namely, relic gravitational waves from inflation.

The most appealing motivation of this work could be the possible direct detection of dark matter in galaxy halos. By focusing on a particular galaxy encounter if the polarization of CMB photons were modified, this would imply that the source responsible of such effect would be the expected dark matter distribution. Therefore, dark matter should exist in concordance with the so-called dark matter paradigm. The detection of those elusive GWs (through their imprint on the $\mathrm{CMB}$ polarization) would provide a smoking-gun signature of the existence of the dark matter. However, the results of our estimates show that the gravitational-wave-induced imprints of galaxy encounters on the CMB are beyond observational capabilities. Nevertheless, there exist additional aspects of the nonlinear dynamics of galaxy mergers which could have implications on potentially observable effects through CMB polarization, namely, gravitational lensing [31] or the socalled Stroskii effect (see Ref. [32] and references therein). This issue will be discussed in a forthcoming paper.

The results reported in this work are similar to those investigated by $[7,33]$ regarding the nonlinear evolution of cosmological structures as sources of gravitational radiation. Future work on this topic should account for the integrated effect of a realistic galaxy distribution. The hopes opened by the upcoming missions to measure ultralow frequency GWs make these investigations worth to pursue.

\section{ACKNOWLEDGMENTS}

The authors thank Alicia Sintes, Miguel Portilla, Juan A. Morales, and José María Ibáñez for useful discussions, and 
VICENT QUILIS et al.

the anonymous referee for the stimulating insights and comments. Research supported by the Spanish Ministerio de Educación y Ciencia (MEC; Grants No. AYA2006-
PHYSICAL REVIEW D 75, 104008 (2007)

02570, AYA2006-08067-C03-01, and FIS2006-06062). V. Q. is a Ramón y Cajal Fellow of the Spanish MEC.
[1] D. E. Holz and S. A. Hughes, Astrophys. J. 629, 15 (2005).

[2] D. Merritt and M. Milosavljevic, Living Rev. Relativity 8, 8 (2005).

[3] D. Merritt, Rept. Prog. Phys. D 69, 2513 (2006).

[4] M. Enoki, K. T. Inoue, M. Nagashima, and N. Sugiyama, Astrophys. J. 615, 19 (2004).

[5] A. Sesana, F. Haardt, P. Madau, and M. Volonteri, Astrophys. J. 623, 23 (2005).

[6] S. Mollerach, D. Harari, and S. Matarrese, Phys. Rev. D 69, 063002 (2004).

[7] C. Carbone, C. Baccigalupi, and S. Matarrese, Phys. Rev. D 73, 063503 (2006).

[8] D. Baskaran, L. P. Grishchuk, and A. G. Polnarev, Phys. Rev. D 74, 083008 (2006).

[9] W. Hu and S. Dodelson, Annu. Rev. Astron. Astrophys. 40, 171 (2002).

[10] M. Bowden et al., Mon. Not. R. Astron. Soc. 349, 321 (2004).

[11] B. Maffei et al., CLOVER: The CMB Polarization Observer, edited by M. Giard, F. Casoli, and F. Paletou (EAS Publications, Tartu, 2005), p. 251.

[12] B. Keating et al., BICEP: A Large Angular Scale CMB Polarimeter, edited by S. Fineschi, Proceedings of the SPIE No. 4843 (2003), p. 284.

[13] A. Toomre and J. Toomre, Astrophys. J. 178, 623 (1972).

[14] A. C. González-García and T. S. van Albada, Mon. Not. R. Astron. Soc. 361, 1030 (2005).

[15] A. C. González-García and T. S. van Albada, Mon. Not. R. Astron. Soc. 361, 1043 (2005).

[16] S. Khochfar and A. Burkert, Mon. Not. R. Astron. Soc.
359, 1379 (2005)

[17] A. Burkert and T. Naab, Mon. Not. R. Astron. Soc. 363, 597 (2005).

[18] A. C. González-García and M. Balcells, Mon. Not. R. Astron. Soc. 357, 753 (2005).

[19] T. Naab, R. Jesseit, and A. Burkert, Mon. Not. R. Astron. Soc. 372, 839 (2006).

[20] W. Jaffe, Mon. Not. R. Astron. Soc. 202, 995 (1983).

[21] K. Kuijken and J. Dubinski, Mon. Not. R. Astron. Soc. 277, 1341 (1995).

[22] V. Springel, N. Yoshida, and S. D. M. White, New Astron. Rev. 6, 79 (2001).

[23] C. W. Misner, K. S. Thorne, and J. A. Wheeler, Gravitation (Freeman, San Francisco, 1973).

[24] L. S. Finn and D. R. Evans, Astrophys. J. 351, 588 (1990).

[25] R. Mönchmeyer, G. Schäfter, E. Müller, and R. E. Kates, Astron. Astrophys. 246, 417 (1991).

[26] H. Dimmelmeier, J. A. Font, and E. Müller, Astron. Astrophys. 393, 523 (2002).

[27] U. Seljak and M. Zaldarriaga, Phys. Rev. Lett. 78, 2054 (1997).

[28] L. Stanghellini, A. C. González-García, and A. Manchado, Astrophys. J. 644, 843 (2006).

[29] T. Naab and A. Burkert, Astrophys. J. 597, 893 (2003).

[30] A. M. Cruise, Mon. Not. R. Astron. Soc. 204, 485 (1983).

[31] W. Hu and M. White, Phys. Rev. D 56, 596 (1997).

[32] J.A. Morales and D. Sáez, Phys. Rev. D 75, 043011 (2007).

[33] V. Quilis, J. M. Ibáñez, and D. Sáez, Astrophys. J. 501, L21 (1998). 